\section{THU0033 RNA PROFILING OF HEALTHY AND RHEUMATOID ARTHRITIS SUBJECTS TREATED WITH TOFACITINIB MONOTHERAPY}

Angela Hadjipanayis, Xing Chen, Julie Lee, Shanrong Zhao, Weidong Zhang, David Martin, Mateusz Maciejewski, Daniel Ziemek, Lori Fitz, Craig Hyde, David von Schack. Pfizer Inc, Cambridge, MA, United States of America

Background: The Janus kinase (JAK) family of tyrosine kinases includes JAK1, JAK2, JAK3 and TYK2 which signals through type I and type II cytokine receptors. Tofacitinib is an oral JAK inhibitor approved for the treatment of rheumatoid arthritis (RA). In cellular settings where JAKs signal in pairs, tofacitinib preferentially inhibits signalling by heterodimeric receptors associated with JAK1 and/or JAK3 and has functional selectivity over JAK2. Next generation sequencing (i.e. RNA sequencing) offers an unbiased analysis of whole transcriptome pharmacology.

Objectives: To profile the transcriptome in whole blood samples collected in a Phase 1 healthy volunteer (HV) study and a Phase 2 study with RA subjects treated with placebo, $5 \mathrm{mg}, 10 \mathrm{mg}$ or $15 \mathrm{mg}$ of tofacitinib monotherapy.

Methods: In the HV study, a total of 93 Paxgene RNA tubes were obtained from 30 subjects collected at screening, at baseline on day 1, and at 2 hours post dosing on day 15. HVs were treated daily with tofacitinib (10 mg BID) for 15 days. In the RA study (NCT00550446), a total of 239 RNA samples were obtained from 25-31 subjects at baseline and day 28 who were treated with tofacitinib $5 \mathrm{mg}$ BID, $10 \mathrm{mg}$ BID or 15 $\mathrm{mg}$ BID daily for 84 days. RNAseq libraries were generated using a polyA based mRNA kit and sequenced to a depth of 40 million pairedend reads. Upstream processing included alignment of sequences, gene quantification and data quality control. The raw reads were normalised using the Trimmed Mean of $M$ values (TMM) method in the edgeR package. Log2 fold-changes (FC) and corresponding false discovery rate (FDR)-adjusted $p$-values (padj) were calculated using the voom function in the Limma package (vers 3.8) in R 3.5.1. In particular, the correlations between baseline and measurements at each visit per patient were estimated by Limma's duplicateCorrelation function. Genes were considered significant after multiple test correction with FDR, or padj $<0.1$ within each study.

Results: Modulation of the JAK-STAT signalling pathways was observed as exemplified by gene expression differences in cytokine-inducible $\mathrm{SH}_{2}$ containing protein $(\mathrm{CISH})$ ( $\mathrm{p}$ value $=4.64 \mathrm{E}-41$ in the HV study and $\mathrm{p}$ value $=1.2 \mathrm{E}-8$ in the RA study) and suppressor of cytokine signalling (SOCS2) protein ( $p$ value $=1.38 \mathrm{E}-18$ in $\mathrm{HV}$ study and $p$ value $=2.27 \mathrm{E}-7$ in the RA study). Gene expression of $\mathrm{CISH}$, a member of the SOCS family, was decreased from its baseline value at day 15 in HV (FC from baseline $=0.18$ ) and at day 28 in RA subjects ( $F C$ from baseline $=0.42$ ) when comparing tofacitinib treatment ( $\mathrm{HV}: 10 \mathrm{mg}$, RA:15 mg) to baseline or placebo, respectively. SOCS2 gene expression was also decreased at day 15 in HV (FC from baseline $=0.40$ ) and at day 28 in RA subjects (FC from baseline $=0.52)$ when comparing tofacitinib treatment $(\mathrm{HV}: 10 \mathrm{mg}$, RA:15 mg) to baseline or placebo, respectively. Other changes observed in the HV study: 301 genes were upregulated and 169 genes were downregulated when comparing 2 hours post dose on day 15 to baseline. In the RA study, 643 genes were upregulated and 801 genes were downregulated when comparing the $15 \mathrm{mg}$ dose to placebo at week 4 . Preliminary pathway level analysis shows overall a broader modulation of cytokine and chemokine signalling pathways by tofacitinib treatment in RA compared to what we observe in HV.

Conclusion: The results of this post hoc analysis demonstrate that tofacitinib induces measureable changes in the modulation of the JAK-STAT signalling pathway as well as effects on inflammatory cytokines and chemokines. The existence of overlapping but also unique JAK-STAT pathway inhibition due to tofacitinib between the HV and RA studies, as well as ongoing work, will provide a better understanding of response/nonresponse to tofacitinib and future drug developments.

Disclosure of Interests: Angela Hadjipanayis Shareholder of: Pfizer Inc, Employee of: Pfizer Inc, Xing Chen Shareholder of: Pfizer Inc, Employee of: Pfizer Inc, Julie Lee Shareholder of: Pfizer Inc, Employee of: Pfizer Inc, Shanrong Zhao Shareholder of: Pfizer Inc, Employee of: Pfizer Inc, Weidong Zhang Shareholder of: Pfizer Inc, Employee of: Pfizer Inc, David Martin Shareholder of: Pfizer Inc, Employee of: Pfizer Inc, Mateusz Maciejewski Shareholder of: Pfizer Inc, Employee of: Pfizer Inc, Daniel Ziemek Shareholder of: Pfizer Inc, Employee of: Pfizer Inc, Lori Fitz Shareholder of: Pfizer Inc, Employee of: Pfizer Inc, Craig Hyde Shareholder of: Pfizer Inc, Employee of: Pfizer Inc, David von Schack Shareholder of: Pfizer Inc, Employee of: Pfizer Inc

DOI: 10.1136/annrheumdis-2019-eular.1928

\section{THU0034 \\ IRAK4 INHIBITION SUPPRESSES TLR7, TLR9, AND SLE SERUM-INDUCED IFNA PRODUCTION IN PRIMARY HUMAN PLASMACYTOID DENDRITIC CELLS}

Angie Hammond, Sean S. Parghi, Stacey Drabic, Nathan Wright, Ethan Grant, James Taylor, Matthew R. Warr. Gilead Sciences, Inc., Foster City, United States of America

Background: A hallmark of lupus is the presence of antinuclear autoantibodies, including those against RNA-protein complexes and doublestranded DNA (dsDNA). ${ }^{1,2}$ FcyR-mediated internalization of these nucleic acid:autoantibody immune complexes results in endosomal activation of TLR7 and TLR9, respectively, and the production of IFN $\alpha$ from plasmacytoid dendritic cells $(\mathrm{pDC})^{3,4}$ The importance of this pathway is underscored by the majority of SLE patients having a peripheral interferonstimulated gene (ISG) signature. ${ }^{5}$ IRAK4 is a serine/threonine kinase at the top of the signaling cascade downstream of TLRs, including TLR7 and TLR9. As such, inhibition of IRAK4 represents a promising therapeutic target for lupus.

Objectives: To investigate the effect of a highly selective IRAK4 inhibitor on IFN $\alpha$ production from pDCs stimulated with TLR7 and TLR9 agonists, SLE serum, and nucleic acid:autoantibody immune complexes.

Methods: Primary human pDCs were precultured with an IRAK4 inhibitor followed by stimulation for 24 hours. Stimulation agents included TLR7 and TLR9 agonists, human SLE serum, and nucleic acid:autoantibody immune complexes. Culture supernatants were then assessed for secretion of IFN $\alpha$ by MSD.

Results: TLR7 and TLR9 stimulation of pDCs resulted in the secretion of IFN $\alpha$ from $\mathrm{pDCs}$ as expected. Treatment with an IRAK4 inhibitor resulted in dose-dependent inhibition of TLR7- and TLR9-induced IFN $\alpha$ with high potency. Human SLE serum was tested to extend findings to physiological SLE-relevant stimuli. Several SLE serum samples, which had positive ELISA titers to the antinuclear antibodies, stimulated production of IFNo in pDCs after 24 hours in culture. No induction of IFN $\alpha$ was observed with healthy volunteer serum samples. Treatment with an IRAK4 inhibitor effectively blocked secretion of IFN $\alpha$ from SLE serum-stimulated pDCs in a dose-dependent manner. Additional antigen (either RNP or dsDNA) was added to SLE samples that yielded titers for autoantibodies but failed to elicit IFN $\alpha$ production. Addition of RNP or dsDNA to anti-RNP and antidsDNA-positive SLE sera induced strong IFN $\alpha$ production, and this response was blocked with IRAK4 inhibition. No induction was seen with SLE serum that was negative for both anti-RNP and anti-dsDNA.

Conclusion: This work demonstrates the effects of IRAK4 inhibition on IFN $\alpha$ production in primary $\mathrm{pDCs}$ downstream of disease-relevant stimuli and highlights the potential for IRAK4 inhibition as a promising treatment for lupus.

\section{REFERENCES:}

[1] Rose T, Dorner T. Best Pract Res Clin Rheumatol. 2017;31:321-333.

[2] Zharkova O, et al. Rheumatology. 2017;56:155-i66.

[3] Barrat FJ, et al. J Exp Med. 2005;202(8):1131-1139.

[4] Means TK, et al. J Clin Invest. 2005;115(2):407-417.

[5] Yao Y, et al. Hum Genomics Proteomics. 2009. 2009(374312):1-16.

Disclosure of Interests: Angie Hammond Grant/research support from: Gilead Sciences, Inc., Employee of: Gilead Sciences, Inc., Sean S. Parghi Grant/research support from: Gilead Sciences, Inc., Employee of: Gilead Sciences, Inc., Stacey Drabic Grant/research support from: Gilead Sciences, Inc., Employee of: Gilead Sciences, Inc., Nathan Wright Shareholder of: Gilead Sciences, Inc., Employee of: Gilead Sciences, Inc. Ethan Grant Shareholder of: Gilead Sciences, Inc., Employee of: Gilead Sciences, Inc., James Taylor Grant/research support from: Gilead Sciences, Inc., Employee of: Gilead Sciences, Inc., Matthew R. Warr Shareholder of: Gilead Sciences, Inc., Employee of: Gilead Sciences, Inc. DOI: 10.1136/annrheumdis-2019-eular.2511

\section{THU0035 DOMINANCE OF DRUG TARGET MECHANISMS IN RHEUMATOID ARTHRITIS}

Jérôme Paul1, Pierre Gramme1, Thibault Helleputte1. DNAlytics, Louvain-laNeuve, Belgium

Background: Biological and Targeted Synthetic Disease-Modifying AntiRheumatic Drugs ( $b$ - and tsDMARDs) have been developed over the years for patients with rheumatoid arthritis (RA). They can be grouped into families of drugs according to their mechanisms of action. Here we focus specifically on anti-TNFs, anti-IL6s, anti-IL1s, T or B Cells inhibitors and JAK inhibitors. There is still a significant proportion of patients 